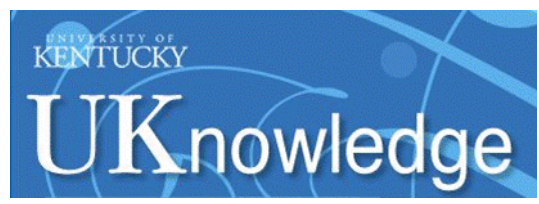

University of Kentucky

UKnowledge

\title{
5-7-2014
}

\section{Ferromagnetic resonance study of eightfold artificial ferromagnetic quasicrystals}

Vinayak Shantaram Bhat

University of Kentucky, vinayak8582@gmail.com

\section{J. Sklenar}

Northwestern University

J. Woods

University of Kentucky

B. Farmer

University of Kentucky

J. B. Ketterson

Northwestern University

See next page for additional authors

Follow this and additional works at: https://uknowledge.uky.edu/physastron_facpub

Part of the Electrical and Computer Engineering Commons, and the Physics Commons

Right click to open a feedback form in a new tab to let us know how this document benefits you.

\section{Repository Citation}

Bhat, Vinayak Shantaram; Sklenar, J.; Woods, J.; Farmer, B.; Ketterson, J. B.; Hastings, Jeffrey Todd; and De Long, Lance E., "Ferromagnetic resonance study of eightfold artificial ferromagnetic quasicrystals" (2014). Physics and Astronomy Faculty Publications. 1.

https://uknowledge.uky.edu/physastron_facpub/1

This Article is brought to you for free and open access by the Physics and Astronomy at UKnowledge. It has been accepted for inclusion in Physics and Astronomy Faculty Publications by an authorized administrator of UKnowledge. For more information, please contact UKnowledge@lsv.uky.edu. 
Ferromagnetic resonance study of eightfold artificial ferromagnetic quasicrystals

Digital Object Identifier (DOI)

http://dx.doi.org/10.1063/1.4859035

Notes/Citation Information

Published in Journal of Applied Physics, v. 115, no. 17C502.

Copyright 2014, American Institute of Physics. This article may be downloaded for personal use only. Any other use requires prior permission of the author and the American Institute of Physics.

This article appeared in Journal of Applied Physics, v. 115, no. 17C502 and may be found at http://dx.doi.org/10.1063/1.4859035.

The copyright holder has granted the permission for posting the article here.

\section{Authors}

Vinayak Shantaram Bhat, J. Sklenar, J. Woods, B. Farmer, J. B. Ketterson, Jeffrey Todd Hastings, and Lance E. De Long 


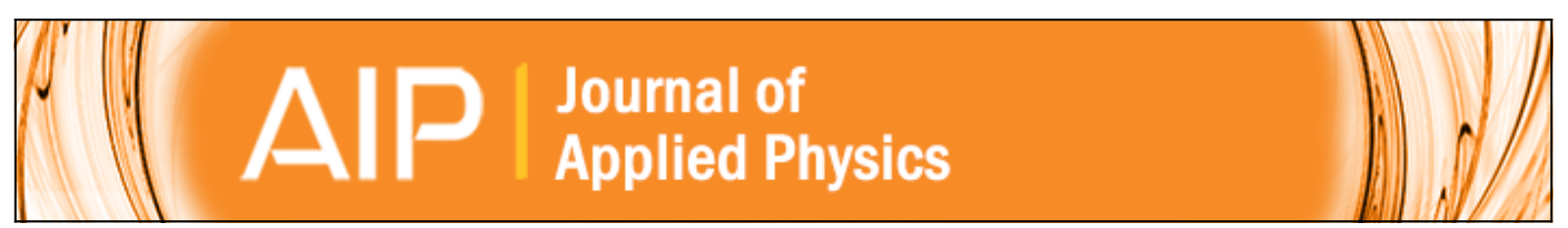

Ferromagnetic resonance study of eightfold artificial ferromagnetic quasicrystals

V. S. Bhat, J. Sklenar, B. Farmer, J. Woods, J. B. Ketterson, J. T. Hastings, and L. E. De Long

Citation: Journal of Applied Physics 115, 17C502 (2014); doi: 10.1063/1.4859035

View online: http://dx.doi.org/10.1063/1.4859035

View Table of Contents: http://scitation.aip.org/content/aip/journal/jap/115/17?ver=pdfcov

Published by the AIP Publishing

\section{Articles you may be interested in}

Nonstochastic magnetic reversal in artificial quasicrystalline spin ice

J. Appl. Phys. 115, 17E133 (2014); 10.1063/1.4869288

Magnetic coupling in asymmetric FeCoV/Ru/FeNi trilayers

J. Appl. Phys. 115, 17D129 (2014); 10.1063/1.4864743

Ferromagnetic resonance investigation of $\mathrm{Py} / \mathrm{Cr}$ multilayer system

J. Appl. Phys. 110, 023908 (2011); 10.1063/1.3613700

Dynamics of the exchange field supplied by Mnlr layers studied by network analyzer ferromagnetic resonance J. Appl. Phys. 106, 063918 (2009); 10.1063/1.3225572

Preparation, structural characterization, and dynamic properties investigation of permalloy antidot arrays J. Appl. Phys. 97, $10 J 506$ (2005); 10.1063/1.1853691

\section{AlP Re-register for Table of Content Alerts}




\title{
Ferromagnetic resonance study of eightfold artificial ferromagnetic quasicrystals
}

\author{
V. S. Bhat, ${ }^{1, a)}$ J. Sklenar, ${ }^{2}$ B. Farmer, ${ }^{1}$ J. Woods, ${ }^{1}$ J. B. Ketterson, ${ }^{2}$ J. T. Hastings, ${ }^{3}$ \\ and L. E. De Long ${ }^{1}$ \\ ${ }^{1}$ Department of Physics and Astronomy, University of Kentucky, Lexington, Kentucky 40506, USA \\ ${ }^{2}$ Department of Physics and Astronomy, Northwestern University, Evanston, Illinois 60208, USA \\ ${ }^{3}$ Department of Electric and Computer Engineering, University of Kentucky, Lexington, Kentucky 40506, USA
}

(Presented 5 November 2013; received 22 September 2013; accepted 11 October 2013; published online 7 January 2014)

We have performed broadband $(10 \mathrm{MHz}-18 \mathrm{GHz})$ and narrowband $(9.7 \mathrm{GHz})$ ferromagnetic resonance (FMR) measurements on permalloy thin films patterned with quasiperiodic Ammann tilings having eightfold rotational symmetry. We observed highly reproducible mode structures in the low-frequency, hysteretic regime in which domain walls and unsaturated magnetization textures exist. A minimum of 10 robust modes were observed in patterned samples, compared to the single uniform mode observed in unpatterned permalloy films. The field dependence and approximate eightfold rotational symmetry of the FMR spectra are in good agreement with micromagnetic simulations that confirm the importance of patterning for controlling static and dynamic magnetic response. (C) 2014 AIP Publishing LLC. [http://dx.doi.org/10.1063/1.4859035]

Ferromagnetic (FM) antidot lattices (ADL) have attracted attention from technological ${ }^{1,2}$ as well as basic science perspectives. $^{2-4}$ Previous studies on FM ADL have mainly focused on periodic arrays (square, rectangular, kagome, etc.). A recent study ${ }^{5}$ of fivefold rotationally symmetric artificial quasicrystals (QCs) (Penrose P2 tilings) patterned into permalloy films displayed static and dynamic properties that differ from those observed for periodic ADL. The ferromagnetic resonance (FMR) spectra for finite Penrose tilings were of particular interest, since they exhibited tenfold rotational symmetry that is only expected for infinite patterns, as well as novel "asymmetric" mode signatures in the hysteretic reversal regime. Herein, we report a FMR study of a second class of permalloy thin films patterned into finite, quasiperiodic Ammann tilings (ATs) that exhibit eightfold rotational symmetry.

We patterned AT by incorporating a deflation algorithm into our electron beam lithography (EBL) software. The algorithm was initialized with an arrangement of squares and rhombi (with interior angles of $45^{\circ}$ and $135^{\circ}$, such as shown in Fig. 1) known as the "Oth generation" AT. Note the tiling can be viewed as a network of film segments of length $d$ and width $W$. A single iteration of the deflation method on a nth generation tiling will produce a $(n+1)$ th generation tiling, as described elsewhere. ${ }^{6,7}$ The new tiling was scaled such that the segment length $d$ remained the same as for the original 0th generation tiling. Two samples of differing generation were prepared with different edge lengths $d$ for the squares and rhombi, and common width $W$ and thickness $t$ (see Table I). Fig. 1 exhibits an SEM image of one write field for sample III136C. Multiple write fields were copied onto a square array in order to increase the (signal-to-noise-ratio) SNR for magnetic measurements.

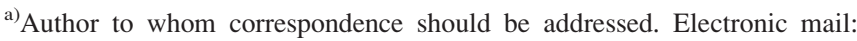
vinayak.bhat@uky.edu.
}

Narrow-band (NB) FMR measurements were carried out at microwave frequency $f=9.7 \mathrm{GHz}$ in applied DC magnetic fields $H \leq 10$ kOe. Broad-band (BB) FMR measurements were performed at room temperature using a meander line method, ${ }^{8}$ in which $f$ can be varied continuously from $10 \mathrm{MHz}$ up to $20 \mathrm{GHz}$. The applied field $\boldsymbol{H}$ could be swept continuously through zero over the range +6 kOe to -6 $\mathrm{kOe}$, and could be rotated within the film plane by an angle $\phi$ between $\boldsymbol{H}$ and the $\mathbf{x}$ - axis (see Fig. 1).

Micromagnetic simulations (MS) were performed to interpret the FMR data by importing a graphic rendition of the Oth generation AT (with $d=1 \mu \mathrm{m}, W=130 \mathrm{~nm}$, and

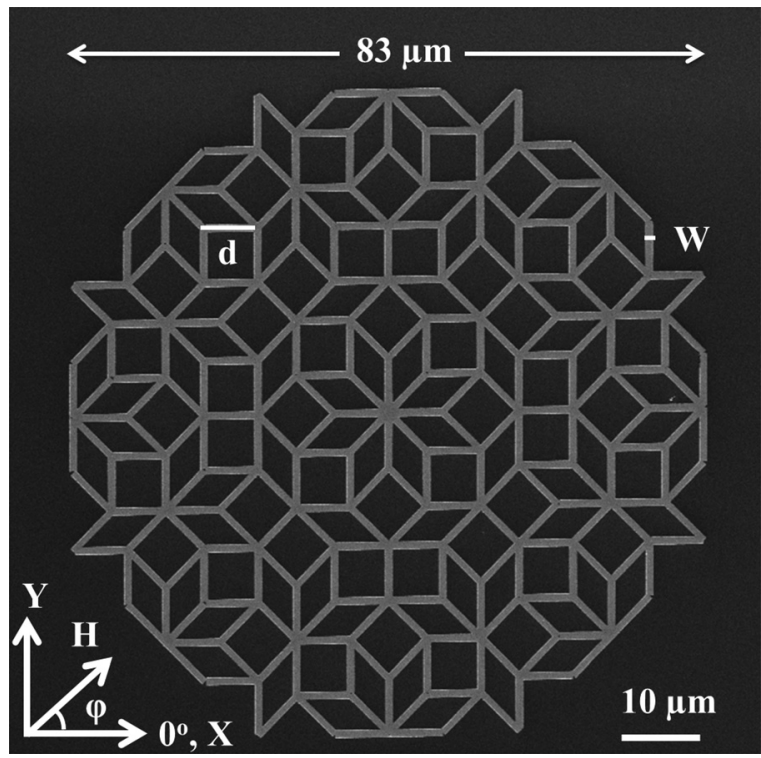

FIG. 1. SEM image of one write field for Sample III136C (see Table I). Non-magnetic substrate and permalloy regions correspond to the dark and the bright areas, respectively. The magnetic field can be rotated by angle $\phi$ with respect to the $\mathbf{x}$-axis, defined to be horizontal. 
TABLE I. Parameters of Ammann tilings.

\begin{tabular}{lcccc}
\hline \hline Sample, generation & $\begin{array}{c}d \\
(\mathrm{~nm})\end{array}$ & $\begin{array}{c}W \\
(\mathrm{~nm})\end{array}$ & $\begin{array}{c}\text { QC apical } \\
\text { width }(\mu \mathrm{m})\end{array}$ & $\begin{array}{c}\text { No. write } \\
\text { fields }\end{array}$ \\
\hline III128D, 4th & 1000 & 130 & 160 & 144 \\
III136C, 0th & 7000 & 912 & 83 & 400 \\
Simulation, 0th & 1000 & 130 & 12.2 & N/A \\
\hline \hline
\end{tabular}

$t=25 \mathrm{~nm}$ ) into OOMMF $\operatorname{code}^{9}$ using a $10 \mathrm{~nm} \times 10 \mathrm{~nm}$ $\times 25 \mathrm{~nm}$ grid (with exchange constant $A=13 \times 10^{-12} \frac{\mathrm{J}}{\mathrm{m}}$ and saturation magnetization $M_{S}=8.6 \times 10^{5} \frac{\mathrm{A}}{\mathrm{m}}$ ). Larger generations were not simulated due to computational limitations. The equilibrium magnetization was calculated for the given applied DC field. A dynamical FMR simulation was conducted by applying a Gaussian magnetic field pulse (of $20 \mathrm{Oe}$ amplitude and 2.5 ps FWHM duration) perpendicular to the film plane, followed by logging 1024 successive values of magnetization vector for each grid pixel at 10 ps time steps. The perpendicular component of magnetization was recorded as a function of $\mathrm{x}, \mathrm{y}$, and time step. The absorbed power for each pixel was obtained by squaring the FFT amplitude, and the signal phase was obtained by calculating the imaginary part of the amplitude. The analysis yields an area map of the magnetization tilt amplitude at the frequency of response. Comparison with the experimental total absorbed power at a given frequency was achieved by integrating local power over all pixels for each frequency step.

Fig. 2 shows the experimental BB FMR spectra for Sample III128D for microwave frequency $f=15.5 \mathrm{GHz}$ and $\boldsymbol{H}$ oriented along $\mathbf{x}$ axis $\left(\phi=0^{\circ}\right)$. We observed five prominent modes symmetrically placed at $H= \pm 1.227, \pm 1.635$, \pm 2.018 , \pm 3.151 , and $\pm 3.763 \mathrm{kOe}$ in both negative and positive field sweeps. The $15.5 \mathrm{GHz}$ spectra also exhibit a lowerfield, "asymmetric" mode that exhibits clear hysteresis: When the DC field was scanned from positive saturation $(H=+6 \mathrm{kOe})$ to negative saturation $(H=-6 \mathrm{kOe})$, only one

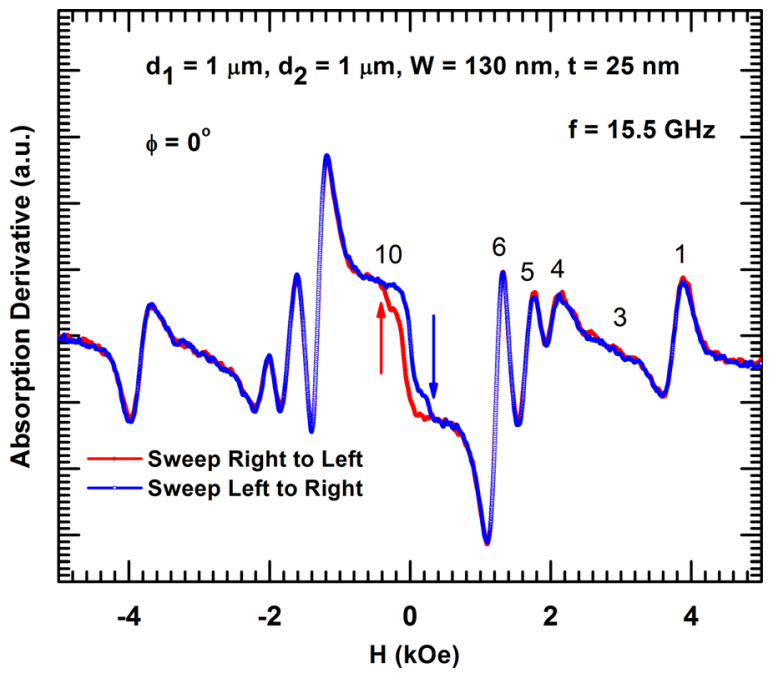

FIG. 2. BB FMR spectra for Sample III128D for frequency $f=15.5 \mathrm{GHz}$. Note reproducible, symmetric FMR spectra for DC fields $|H|>1$ kOe; asymmetric modes only occur on one side of origin near $|H|=0.3 \mathrm{kOe}$ (branch $\mathbf{1 0}$ in Fig. 3). The blue arrow marks an asymmetric mode signature for negative sweep and the red arrow marks the same mode for positive sweep. Numbers denote mode branches.

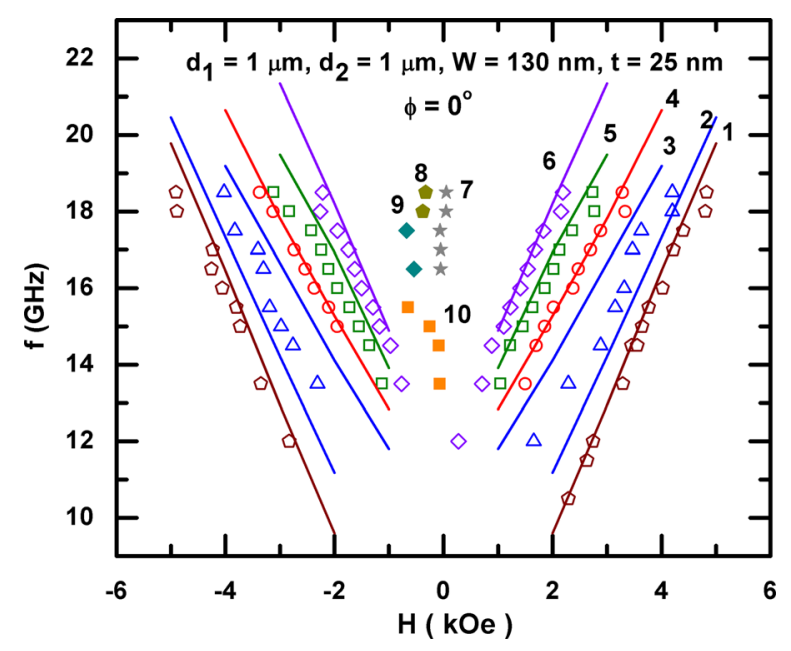

FIG. 3. BB FMR frequency $f$ vs. DC field $H$ for Sample III128D. Scattered points and solid lines represent experimental data and simulations, respectively. Numbers denote mode branches. Note the low-field asymmetric mode branches $7-10$ in the hysteretic regime.

mode signature was observed at $H=-0.33 \mathrm{kOe}$, on the $\boldsymbol{n e g}$ ative side of zero. On the other hand, when magnetic field was swept from negative saturation $(H=-6 \mathrm{kOe})$, a corresponding mode signature was observed only at $H=+0.29$ $\mathrm{kOe}$, on the positive side of zero. MS indicate the presence of domain walls and complex switching behavior of the permalloy segments in the hysteretic regime give rise to the asymmetric mode near zero field. The reproducibility of the mode structure over a wide range of fields in Fig. 2 is evidently due to shape anisotropy induced by patterning. ${ }^{5}$

Fig. 3 shows the experimental (4th generation) BB FMR data and MS results (0th generation) for the frequency vs. field dispersion relations for ten mode branches. The simulated FMR mode branches 1, 4, 5, and 6 are in very good agreement with experiments; whereas the experimental BB FMR data only reveal one broad mode located in between the simulated branches 2 and 3 .

Figs. 4(a)-4(c) show simulated local power maps corresponding to $f=16.5 \mathrm{GHz}(H=4 \mathrm{kOe}$; branch 1 in Fig. 3$)$, $f=14.5 \mathrm{GHz} \quad(H=2 \mathrm{kOe}$; branch 3 in Fig. 3), and $f=18.4 \mathrm{GHz}(H=2 \mathrm{kOe}$; branch 6 in Fig. 3). The dynamic simulations, done only for the 0th generation AT, suggest FMR modes at $|H|=1.227,1.63$, and $3.763 \mathrm{kOe}$ (see Fig. 2) are due to power absorption by permalloy segments at an angle $\theta=0^{\circ}$ and $180^{\circ}$ (branch 6 in Fig. 3), $\pm 45^{\circ}$ (branch 5 in Fig. 3), and $\pm 90^{\circ}$ (branch 1 in Fig. 3), respectively. Here, $\theta$ is defined to be the angle between a segment's long axis and the ${ }^{10}$ direction of the film. A FMR mode at $|H|=2.018$ $\mathrm{kOe}$ is due to power absorption in both $\theta= \pm 45^{\circ}$ and $\pm 90^{\circ}$ segments (branch 4 in Fig. 3). A weaker mode at $|H|=$ $3.151 \mathrm{kOe}$ may be a second harmonic of the mode responsible for power absorption in both $\pm 45^{\circ}$ and $\pm 90^{\circ}$ segments (overlapping branch 2 and/or 3 in Fig. 3).

Fig. 5 shows NB FMR spectra for different field angles, $0^{\circ} \leq \phi \leq 90^{\circ}$, and $f=9.7 \mathrm{GHz}$ for Sample III136C. For $\phi=0^{\circ}$, we observed FMR peaks at $|H|=1.18,1.07,0.929$, 0.809 , and $0.682 \mathrm{kOe}$. Fig. 5 shows the FMR spectra exhibit near-eightfold rotational symmetry, consistent with the 


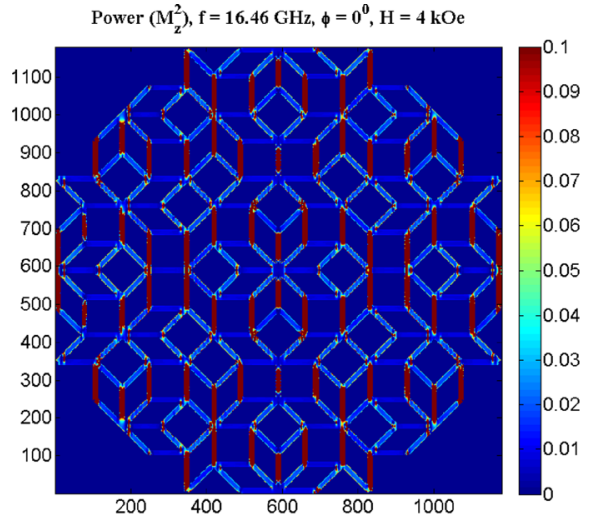

(a)

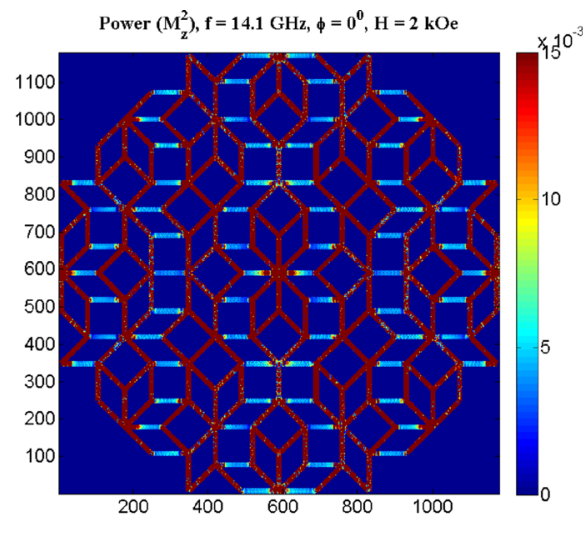

(b)

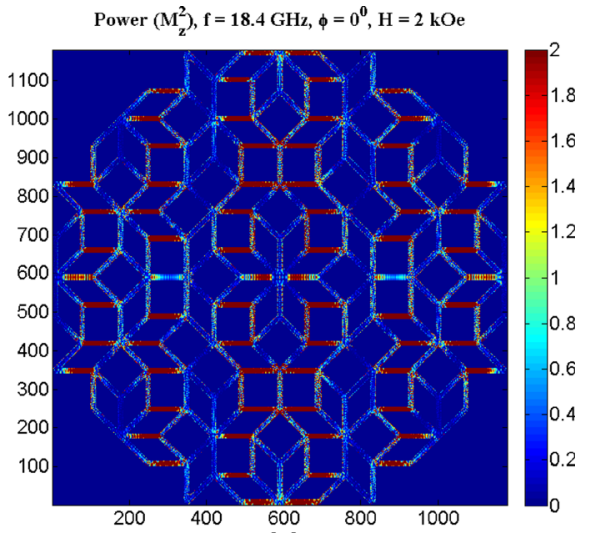

(c)

FIG. 4. (a) Simulated OOMMF power absorption maps for a 0th generation AT with $d=1 \mu \mathrm{m}, W=130 \mathrm{~nm}$, and $t=25 \mathrm{~nm}$, field angle $\phi=0^{\circ}$, frequency $f=16.5 \mathrm{GHz}$, and applied DC field $H=4 \mathrm{kOe}$. Color scale indicates the squared magnetization tipping amplitude (red is greatest). This power map corresponds to branch 1 (brown) in Fig. 3. (b) Power map for $f=14.1 \mathrm{GHz}, H=2$ kOe corresponding to branch 3 (blue) in Fig. 3. (c). Power map for $f=18.4 \mathrm{GHz}$, $H=2$ kOe corresponding to branch 6 (purple) in Fig. 3 .

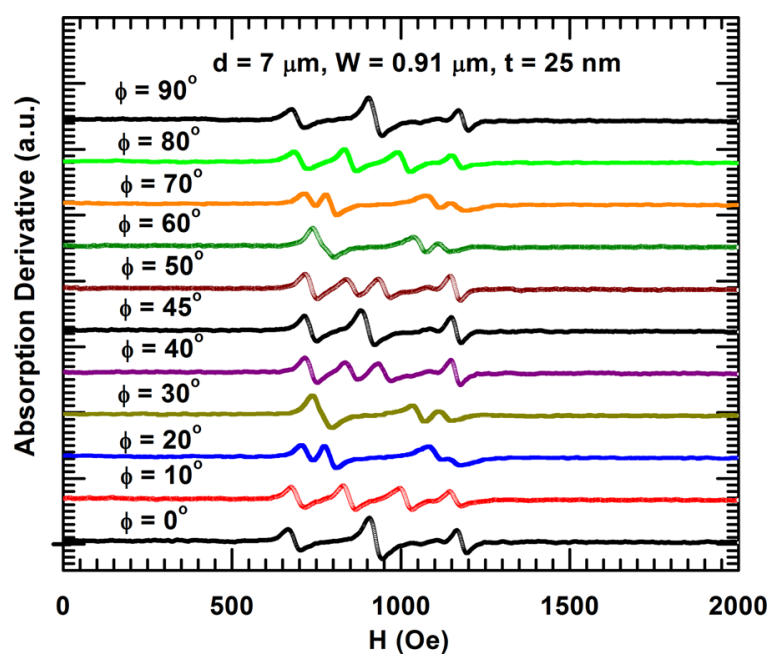

FIG. 5. NB FMR absorption derivative versus applied DC magnetic field $H$ of Sample III136C at $T=300 \mathrm{~K}$ for frequency $f=9.7 \mathrm{GHz}$. Colors correspond to different angles $0^{\circ} \leq \phi \leq 90^{\circ}$. Note that $\phi=0^{\circ}, 45^{\circ}$, and $90^{\circ}$ are reproduced, which is indicative of eightfold rotational symmetry.

geometry of the AT (Fig. 1). Small departures (1\%-7\%) of the resonance field data from eightfold symmetry is under investigation.

We have fabricated novel artificial FM quasicrystals that are eightfold rotationally symmetric. NB FMR data display eightfold rotationally symmetric FMR absorption peaks. Our FMR data include many more modes than were seen in a previous study of a periodic antidot lattice, ${ }^{10}$ due to the complex topology and lower symmetry of the AT. BB FMR experiments exhibit symmetrically placed modes in the near saturated regime and novel asymmetric modes in the hysteretic regime, similar to recent observations of Penrose P2 tilings. ${ }^{5}$

Research at the University of Kentucky was supported by U.S. DOE Grant No. DE-FG02-97ER45653 and the U.K. Center for Advanced Materials (supported by U.S. NSF Grant No. EPS-0814194).

${ }^{1}$ R. Cowburn, A. Adeyeye, and J. Bland, Appl. Phys. Lett. 70, 2309 (1997). ${ }^{2}$ B. Lenk et al., Phys. Rep. 507, 107 (2011).

${ }^{3}$ C. Yu, M. J. Pechan, and G. J. Mankey, Appl. Phys. Lett. 83, 3948 (2003). ${ }^{4}$ W. Branford et al., Science 335, 1597 (2012).

${ }^{5}$ V. S. Bhat et al., Phys. Rev. Lett. 111, 077201 (2013).

${ }^{6} \mathrm{See}$ http://demonstrations.wolfram.com/AmmannTiles/ for instructions on how to construct the Ammann tiling.

${ }^{7}$ F. P. M. Beenker, "Algebraic theory of non-periodic tilings of the plane by two simple building blocks: A square and a rhombus," TH-Report No. 82WSK04, Eindhoven University of Technology, 1982.

${ }^{8}$ C.-C. Tsai et al., Rev. Sci. Instrum. 80, 023904 (2009).

${ }^{9}$ M. J. Donahue and D. G. Porter, OOMMF User's Guide (NIST, 1999).

${ }^{10}$ V. Bhat et al., Physica C 479, 83 (2012). 